\title{
Copolymer-Based Photonic Crystal Sensor for Discriminative Detection of Liquid Benzene, Toluene, Ethylbenzene, and Xylene
}

Donghui Kou, Wei Ma* Shufen Zhang and Bingtao Tang

State Key Laboratory of Fine Chemicals, Dalian University of Technology, Dalian, Liaoning 116023, P. R. China.

E-mail: weima@dlut.edu.cn; Tel: +86-411-84986506.

The first-order reflection wavelength of a 1DPC can be predicted by Bragg's law (1) and the maximum Bragg peak intensity is given by the following expression (2):

$\lambda_{\max }=2\left(n_{l} d_{l}+n_{h} d_{h}\right)$

$\mathrm{R}=\left(\frac{1-Y}{1+Y}\right)^{2} \times 100 \%, \quad Y=\left(\frac{n_{h}}{n_{l}}\right)^{N-1} \frac{n_{h}^{2}}{n_{s}}$

$\lambda_{\max }$ is the position of the first-order refraction wavelength, $d_{h}$ and $d_{l}$ are the thickness of the highand low-refractive-index regions, $n_{s}$ is the refractive index of the substrate, $n_{h}$ and $n_{l}$ are the refractive indices of the high- and low-index regions, respectively, $N$ is the number of periods of the 1DPCs. 


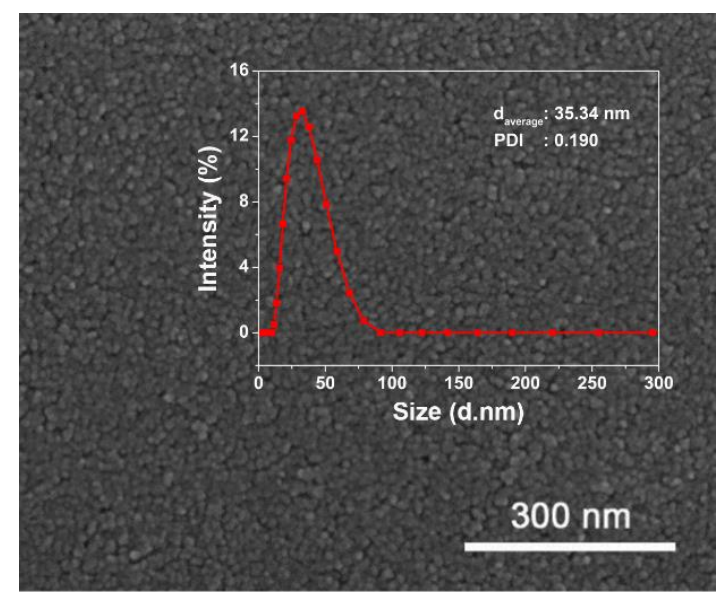

Figure S1. SEM image and particle size distribution (inset) of $\mathrm{TiO}_{2}$ nanoparticles.
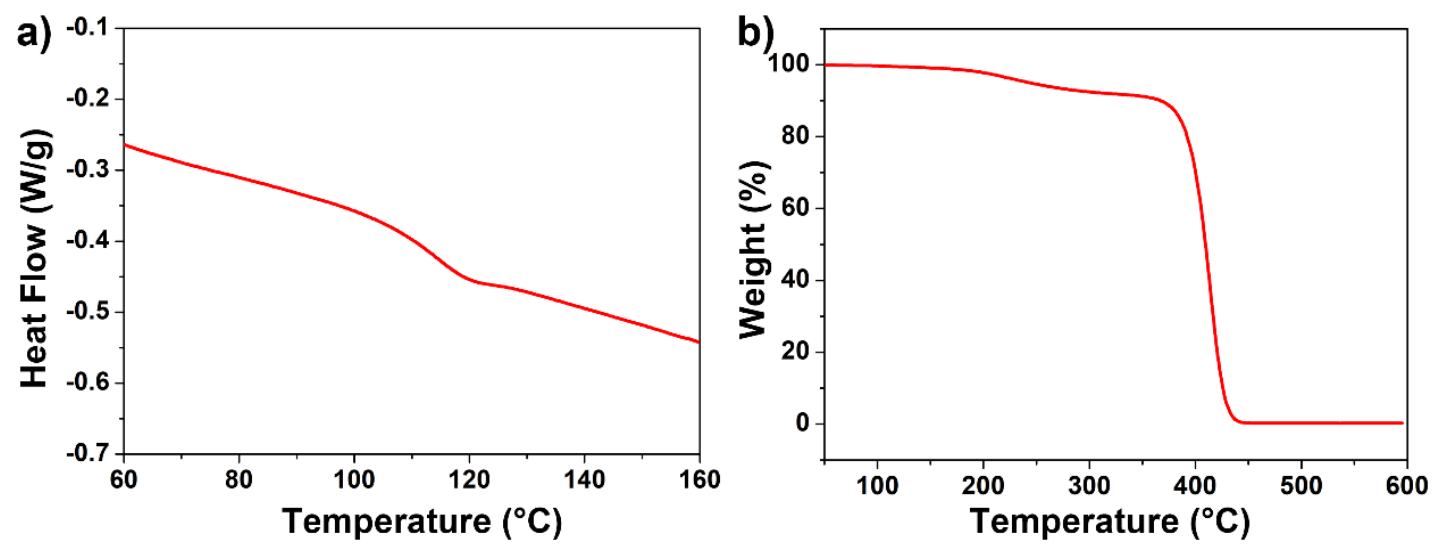

Figure S2. a) DSC curve and b) TGA thermogram of P(St-EGDMA-AA).

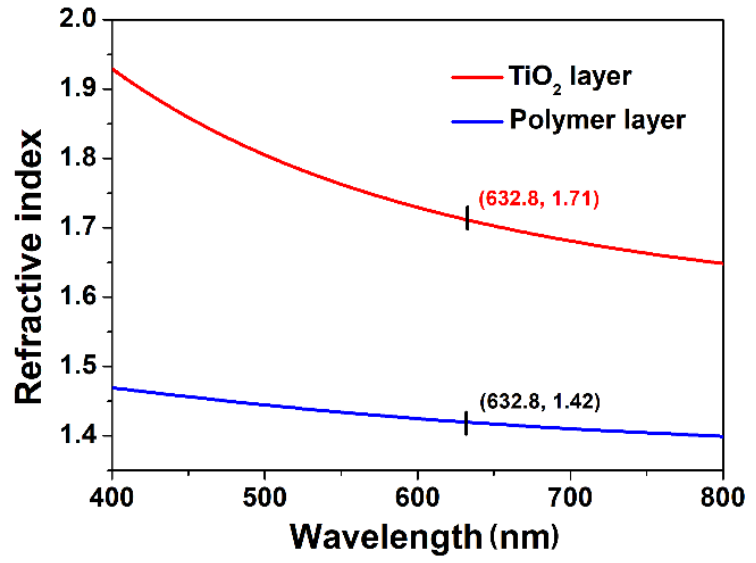

Figure S3. Refractive indexes of $\mathrm{P}(\mathrm{St}-\mathrm{EGDMA}-\mathrm{AA})$ and $\mathrm{TiO}_{2}$ film. 

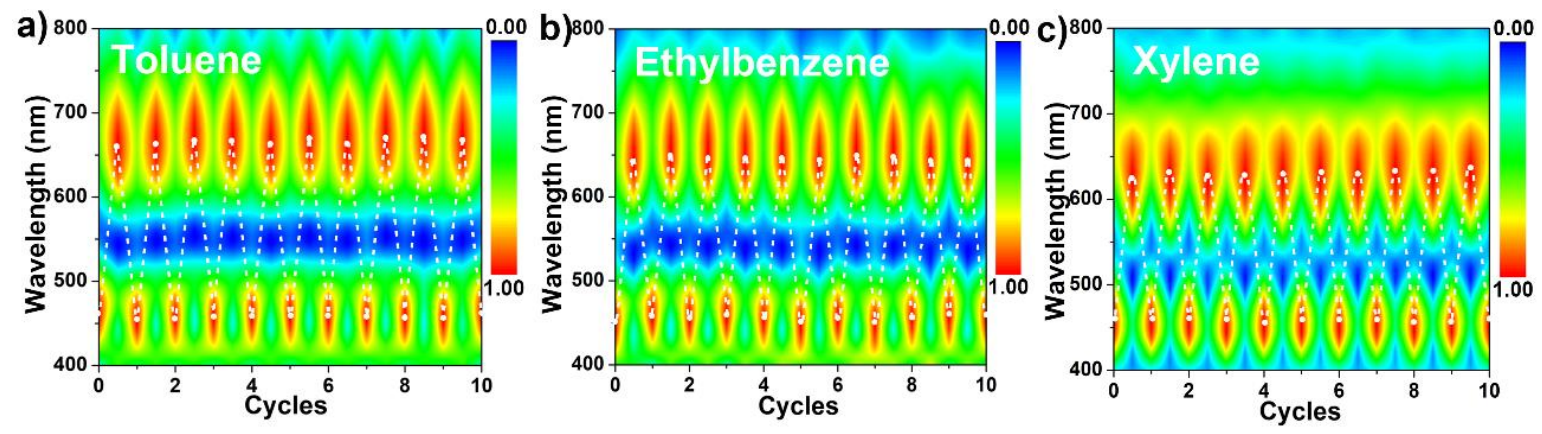

Figure S4. Contour plots of recycle detection test of the sensor in liquid a) toluene, b) ethylbenzene and c) xylene.

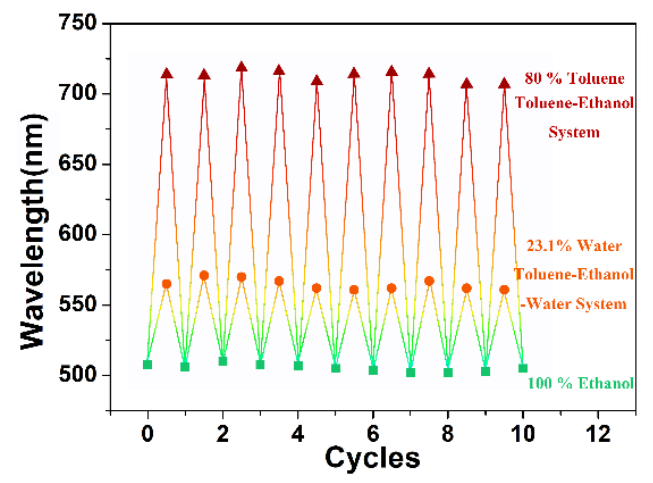

Figure S5. Recycle detection test of the sensor in toluene-ethanol binary mixtures ( $80 \%$ toluene) and toluene-ethanol-water ternary mixtures (7.7\% toluene, $69.2 \%$ ethanol and $23.1 \%$ water).

Video S1. Response process of the PC sensor when immersed in liquid benzene.

Video S2. Response process of the PC sensor when immersed in liquid toulene.

Video S3. Response process of the PC sensor when immersed in liquid ethylbenzene.

Video S4. Response process of the PC sensor when immersed in liquid xylene. 